\title{
Synthesis and antimycobacterial activities of certain trifluoromethyl-aminoquinoline derivatives
}

\author{
Alka Mital, ${ }^{a, *}$ Villendra Singh Negi, ${ }^{a}$ and Uma Ramachandran ${ }^{b}$ \\ ${ }^{a}$ Department of Pharmaceutical Technology, National Institute of Pharmaceutical Education and \\ Research (NIPER), Sector-67, S.A.S. Nagar, Punjab-160 062, India \\ ${ }^{b}$ Orchid Chemicals \& Pharmaceuticals Ltd., 476/14, Old Mahabalipuram Road \\ Sholinganallur, Chennai - 600 119, India \\ E-mail: alkamital@gmail.com
}

\begin{abstract}
A number of 4-amino substituted 2,8-bis(trifluoromethyl)quinoline derivatives were synthesized and evaluated for their in vitro antimycobacterial activity against Mycobacterium tuberculosis (M. $t b)$ strain H37Rv. Preliminary results indicated that most of the compounds demonstrated better in vitro antimycobacterial activity, and are comparable to the first line antituberculosis drugs. The most effective compound 1 of the series has a MIC of $3.13 \mu \mathrm{g} / \mathrm{ml}, \mathrm{IC}_{50}$ value of 3.9. The objective of our study is to generate new leads and to optimize their structure to display the potent efficacy.
\end{abstract}

Keywords: Tuberculosis, antimycobacterial activity, drug-resistant tuberculosis, 2,8bis(trifluoromethyl) amino quinoline derivatives, antituberculosis activity

\section{Introduction}

Tuberculosis (TB), one of the most common infectious diseases, continues to be a major cause of morbidity and mortality all over the world. About $32 \%$ of the world's population is currently infected with TB. Every year, approximately eight to nine million of these infected people develop clinical pulmonary tuberculosis leading to nearly three million annual deaths ${ }^{1,2}$. If the present trend continues, tuberculosis is likely to claim more than 30 million lives within the next decade.

The first-line drugs currently used for the treatment of TB are streptomycin, isoniazid (INH), ethambutol, pyrazinamide and rifampicin (RMP) ${ }^{3}$. Moreover, the emergence of multidrug resistant (MDR) strains of $M$. $t b$, which are insensitive to one or more of the first-line drugs, isoniazid and rifampicin, has further worsened the situation ${ }^{4}$. Furthermore, the association of TB 
and HIV infections has caused an urgent need in search of alternative chemotherapeutics for $M$. $t b$ infection ${ }^{5,6}$.

It is well known that no new antituberculosis drug has been discovered in the last 40 years. Therefore, in order to control the rapid spread of tuberculosis, there is an urgent need for new anti-TB drugs with unique modes of action and improved properties such as enhanced activity against MDR strains, reduced toxicity and shortened duration of therapy ${ }^{7}$. During the past decade, several of the fluoroquinoline antibacterial drugs have been examined as potential chemotherapeutics for $M$. $t b$ infection ${ }^{8-10}$. However, the structural modification of the fluoroquinolones with respect to their optimum anti-TB activity has not been thoroughly explored.

Our research efforts towards the development of novel antituberculosis agents are in the direction of discovering new classes of compounds, which are structurally different from known anti-tuberculosis drugs ${ }^{11}$. It is expected that this approach will offer analogues, which may be effective against drug-resistant strains of $M$. $t b$.

In the continuation of our search, this preliminary communication describes the synthesis and primary screening of 4-amino substituted 2,8-bis(trifluoromethyl)quinoline derivatives against $M$. $t b$ strain $\mathrm{H} 37 \mathrm{Rv}$.

The preparation of 4-amino substituted 2,8-bis(trifluoromethyl)quinoline derivatives is outlined in Scheme 1. All the 4-amino derivatives were prepared in a single step reaction. The starting material 2,8-bis(trifluoromethyl)-4-chloroquinoline was synthesized by known literature methods ${ }^{12}$; 4-aminoquinolines 1-4 containing metabolically inert basic side-chain ( $N, N^{\prime}-$ diethylaminoalkyl group) of altered length such as tert-butylamino, piperidino, pyrrolidino, or morpholino were synthesized according to the depicted scheme. Compounds were then purified by column chromatography. Bisquinolines 5-10 were synthesized via a displacement reaction with 2,8-bis(trifluoromethyl)-4-chloroquinoline, alkanediamine, and triethylamine in a 2:1:2 ratio using $N$-methylpyrrolidinone as solvent. Compounds were isolated by adding water and ethyl acetate or diethyl ether to the cooled reaction mixtures which initiated product precipitation and dissolved any unreacted starting materials. 

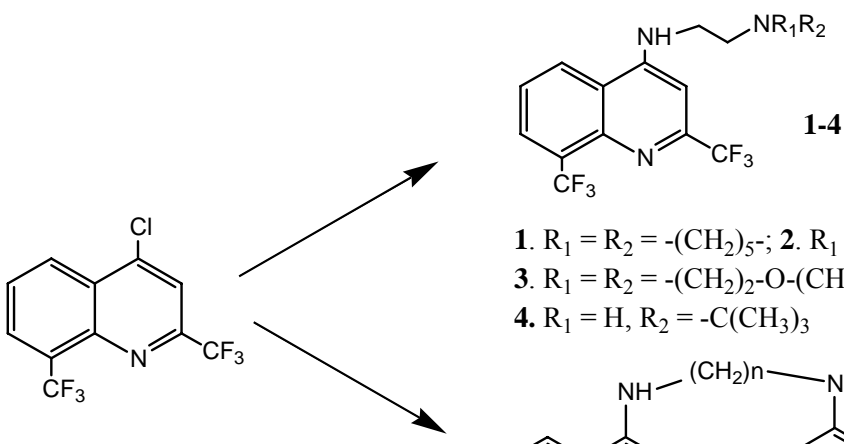

1. $\mathrm{R}_{1}=\mathrm{R}_{2}=-\left(\mathrm{CH}_{2}\right)_{5^{-}} ; 2 \cdot \mathrm{R}_{1}=\mathrm{R}_{2}=-\left(\mathrm{CH}_{2}\right)_{4}$;

3. $\mathrm{R}_{1}=\mathrm{R}_{2}=-\left(\mathrm{CH}_{2}\right)_{2}-\mathrm{O}-\left(\mathrm{CH}_{2}\right)_{2}$;

4. $\mathrm{R}_{1}=\mathrm{H}, \mathrm{R}_{2}=-\mathrm{C}\left(\mathrm{CH}_{3}\right)_{3}$

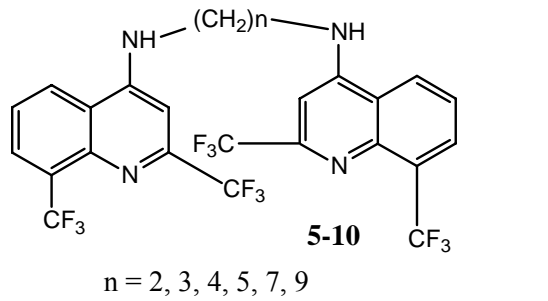

Scheme 1. Synthesis of 4-amino substituted 2,8-bis(trifluoromethyl)quinoline derivatives.

\section{Results and Discussion}

All of the synthesized compounds were primarily screened against Mycobacterium tuberculosis strain H37Rv (ATCC 27294) using the Microplate Alamar Blue Assay (MABA) ${ }^{13}$ at the single concentration, $6.25 \mu \mathrm{g} / \mathrm{mL}$. Compounds were also tested in the BACTEC 460 radiometric system ${ }^{14}$, and the activities are expressed as Minimum Inhibitory Concentration (MIC, $\mu \mathrm{g} / \mathrm{mL}$ ) as summarized in Table 1. The MIC is defined as the lowest concentration effecting a reduction in fluorescence of $90 \%$ relative to controls. Compound 1 demonstrating $\geq 95 \%$ inhibition at $6.25 \mu \mathrm{g} / \mathrm{mL}$ in the initial screen was retested in the broth microdilution assay at the lower concentrations to determine the actual MIC value. Activity is designated by a + in the column labeled Activity. Compound $\mathbf{1}$ was further screened by serial dilution to assess cytotoxicity to a VERO cell line, to determine the selectivity index (SI), defined as the ratio of the measured $\mathrm{IC}_{50}$ in VERO cells to the MIC described above. Compound 1 showed a MIC value of $3.13 \mu \mathrm{g} / \mathrm{ml}$, $\mathrm{IC}_{50}$ value of 3.9 and SI of 1.25 .

The present study on the synthesis and evaluation of 4-amino substituted 2,8bis(trifluoromethyl)quinolines has established the discovery of new types of quinoline analogues with significant and promising anti-tuberculosis activity against $M$. $t b$ H37Rv. Biological studies clearly indicate that the presence of diaminoalkyl chain and trifluoromethyl groups in the 2 and 8 positions of the quinoline ring gives useful biological activity. These effective derivatives are ideally suited for further modifications to obtain more efficacious antimycobacterial compounds. 
Table 1. In vitro antimycobacterial activity data of 4-amino substituted 2,8bis(trifluoromethyl)quinoline derivatives, expressed as $\mathrm{MIC}(\mathrm{ug} / \mathrm{mL})^{\mathrm{a}}$ against drug-sensitive strain of M. tb H37Rv

\begin{tabular}{|c|c|c|c|c|c|}
\hline Sample No. & Structure & Assay & MIC (ug/mL) & \% Inhibition & Activity \\
\hline 1 & & Alamar & $<6.25$ & 98 & + \\
\hline 2 & & Alamar & $>6.25$ & 62 & - \\
\hline 3 & & Alamar & $>6.25$ & 49 & - \\
\hline 4 & & Alamar & $>6.25$ & 4 & - \\
\hline 5 & & Alamar & $>6.25$ & 46 & - \\
\hline 6 & & Alamar & $>6.25$ & 78 & - \\
\hline 7 & & Alamar & $>6.25$ & 89 & - \\
\hline 8 & & Alamar & $>6.25$ & 10 & - \\
\hline 9 & & Alamar & $>6.25$ & 14 & - \\
\hline 10 & & Alamar & $>6.25$ & 20 & - \\
\hline
\end{tabular}

${ }^{\mathrm{a}} \mathrm{MIC}$ is defined as the lowest concentration inhibiting $90 \%$ of the inoculum relative to controls. 
Amongst the tested compounds, 2,8-bis(trifluoromethyl)quinoline 1 with 2-piperidin-1-yl-ethyl amine side-chain exhibited highest inhibitory activity (98\%) at a concentration of $<6.25 \mu \mathrm{g} / \mathrm{mL}$. At the same time bisquinoline 6, with 1, 7-diamino heptane chain also exhibited potent activity $(78 \%)$ at a concentration of $>6.25 \mu \mathrm{g} / \mathrm{mL}$. Activity was further increased in compound 7 to $89 \%$ with increase in the chain length. Compounds 2, 3 with 2-pyrrolidin-1-yl-ethyl amine and 2morphplin-4-yl-ethyl amine side-chain also exhibited 62 and $49 \%$ inhibition at the same concentration. Further investigations on the structural modifications are currently underway and results will be reported in due course.

\section{Experimental Section}

General Procedures. Melting points were recorded on a Buchi capillary melting point apparatus and are uncorrected. Infrared (IR) spectra were recorded on a Nicolet Impact-410 FTIR spectrometer. ${ }^{1} \mathrm{H}$ spectra were recorded on a $300 \mathrm{MHz}$ Bruker FT-NMR spectrometer in $\mathrm{CDCl}_{3}$ or DMSO- $\mathrm{d}_{6}$ solution. The chemical shifts are reported in $\delta(\mathrm{ppm})$ relative to internal standard tetramethylsilane (TMS) and coupling constants $\mathrm{J}$ are given in Hz. Mass spectrometry was conducted using GCMS (Shimadzu QP 5000 spectrometer) auto sampler/direct injection (EI/CI) or LCMS (Finnigan Mat LCQ spectrometer) (APCI/ESI). Elemental analyses were recorded on Elementar Vario EL analyzer. All chromatographic purifications were performed with silica gel 60 (230-400 mesh), whereas all TLC development was done on silica gel coated (Merck Kiesel $60 \mathrm{~F} 254,0.2 \mathrm{~mm}$ thickness) plates. All chemicals were purchased from Aldrich Chemical Company (USA) and were used as received unless otherwise noted. Solvents used for the chemical synthesis were of laboratory and analytical grade, and were used without further purification.

General method for the synthesis of the derivatives 1-4. To a solution of 2,8bis(trifluoromethyl)-4-chloroquinoline $(5 \mathrm{mmol} ; 1.5 \mathrm{~g})$ in ethanol $(10 \mathrm{ml})$ was added amine $(7$ $\mathrm{mmol}$ ). The reaction was heated to reflux for $18 \mathrm{~h}$, after which time the solvent was removed in vacuum. The resultant solid was purified by column chromatography (silica gel) using hexane/ethyl acetate (9:1) as eluent to give the required products $\mathbf{1 - 4}$ as a yellow viscous solid. The final products were recrystallized with ethanol.

(2,8-Bis-trifluoromethylquinolin-4-yl)-(2-piperidin-1-yl-ethyl) amine (1). Yield: 65 \%, m.p. $84^{\circ} \mathrm{C}$; IR (Neat, $\mathrm{cm}^{-1}$ ): 3333, 2927, 2819, 1599, 1551, 1445, 1317, 1118; LCMS/MS: m/z 392 $(\mathrm{M}+1) ;{ }^{1} \mathrm{H}$ NMR $\left(\mathrm{CDCl}_{3}\right): \delta 8.10\left(\mathrm{~d}, \mathrm{~J}=8.5 \mathrm{~Hz}, 1 \mathrm{H},-\mathrm{C} \underline{\mathrm{H}}-\mathrm{C}_{-}-\mathrm{CF}_{3}\right), 8.04(\mathrm{~d}, \mathrm{~J}=7.3 \mathrm{~Hz}, 1 \mathrm{H}), 7.55(\mathrm{t}$, $\mathrm{J}=7.9 \mathrm{~Hz}, 1 \mathrm{H}), 6.73(\mathrm{~s}, 1 \mathrm{H}), 6.55(\mathrm{~s}, 1 \mathrm{H},-\mathrm{N} \underline{\mathrm{H}}), 3.45-3.39$ (m, 2H, -HNC $\left.\underline{H}_{2}-\right), 2.99-2.95$ (m, 2H, $\left.\underline{\mathrm{CH}}_{2} \mathrm{~N}-\right), 2.69$ (m, 4H, - $\left.\underline{\mathrm{C}}_{2} \mathrm{NC}_{2}-\right), 1.88\left(\mathrm{~m}, 4 \mathrm{H},-\mathrm{C}_{2}-\mathrm{C}_{2}-\right), 1.37-1.26(\mathrm{~m}, 2 \mathrm{H})$; Anal. Calcd for $\mathrm{C}_{18} \mathrm{H}_{19} \mathrm{~F}_{6} \mathrm{~N}_{3}$ (391.35): C, $55.24 \%$;, $4.89 \%$;, $10.74 \%$. Found: $\mathrm{C}, 55.20 \%$; $4.80 \%$; , $10.72 \%$.

(2,8-Bis-trifluoromethylquinolin-4-yl)-(2-pyrrolidin-1-yl-ethyl) amine (2). Yield: $52 \%$, m.p. $95^{\circ} \mathrm{C}$; IR (Neat, $\mathrm{cm}^{-1}$ ): 3332, 2955, 2925, 2832, 1599, 1548, 1442, 1312, 1183; LCMS/MS: 
$\mathrm{m} / \mathrm{z} 378(\mathrm{M}+1) ;{ }^{1} \mathrm{H} \mathrm{NMR}\left(\mathrm{CDCl}_{3}\right): \delta 8.05\left(\mathrm{~d}, \mathrm{~J}=7.3 \mathrm{~Hz}, 1 \mathrm{H},-\mathrm{C} \underline{\mathrm{H}}-\mathrm{C}-\mathrm{CF}_{3}\right), 7.98(\mathrm{~d}, \mathrm{~J}=8.5 \mathrm{~Hz}, 1 \mathrm{H})$, $7.56(\mathrm{t}, \mathrm{J}=7.9 \mathrm{~Hz}, 1 \mathrm{H}), 6.73(\mathrm{~s}, 1 \mathrm{H}), 6.57(\mathrm{~s}, 1 \mathrm{H},-\mathrm{NH}), 3.37-3.32\left(\mathrm{~m}, 2 \mathrm{H},-\mathrm{HNC}_{2}-\right)$, 2.78-2.74 (m, $\left.2 \mathrm{H},-\underline{\mathrm{CH}}_{2} \mathrm{~N}-\right), 2.49$ (m, 4H, $\left.-\underline{\mathrm{CH}}_{2} \mathrm{NC}_{2}-\right), 1.68-1.52$ (m, 4H, - $\underline{\mathrm{C}}_{2}-\underline{\mathrm{C}}_{2}-$ ); Anal. Calcd for $\mathrm{C}_{17} \mathrm{H}_{17} \mathrm{~F}_{6} \mathrm{~N}_{3}$ (377.13): C, $54.11 \%$; H, $4.54 \%$; N, $11.14 \%$. Found: C, $54.10 \%$; H, 4.50\%; N, $11.10 \%$.

(2,8-Bis-trifluoromethylquinolin-4-yl)-(2-morpholin-4-yl-ethyl) amine (3). Yield: 60 \%, m.p. $95^{\circ} \mathrm{C}$; IR (Neat, $\mathrm{cm}^{-1}$ ): 3370, 2953, 2898, 2853, 1598, 1546, 1491, 1441, 1360,1183; LCMS/MS: $\mathrm{m} / \mathrm{z} 394(\mathrm{M}+1) ;{ }^{1} \mathrm{H}$ NMR $\left(\mathrm{CDCl}_{3}\right): \delta 8.07$ (d, J=7.2 Hz, 1H, -Cㅡ- $\left.-\mathrm{C}-\mathrm{CF}_{3}\right), 7.98$ (d, $\mathrm{J}=8.4 \mathrm{~Hz}, 1 \mathrm{H}), 7.58(\mathrm{t}, \mathrm{J}=7.8 \mathrm{~Hz}, 1 \mathrm{H}), 6.75(\mathrm{~s}, 1 \mathrm{H}), 6.32(\mathrm{~s}, 1 \mathrm{H},-\mathrm{N} \underline{\mathrm{H}}), 3.80-3.77\left(\mathrm{~m}, 4 \mathrm{H},-\left(\mathrm{C}_{2}\right)-\right.$ O-( $\left.\left.\underline{\mathrm{CH}}_{2}\right)-\right)$, 3.43-3.38 (m, 2H, - $\left.\mathrm{HNC}_{2}{ }_{2}\right), 2.86-2.82\left(\mathrm{~m}, 2 \mathrm{H},-\underline{\mathrm{C}}_{2} \mathrm{~N}-\right), 2.60-2.58$ (m, 4H, $\underline{\mathrm{CH}}_{2} \mathrm{NC}_{2}-$ ); Anal. Calcd for $\mathrm{C}_{17} \mathrm{H}_{17} \mathrm{~F}_{6} \mathrm{~N}_{3} \mathrm{O}$ (393.33): C, $51.91 \%$;, $4.36 \%$; N, $10.68 \%$. Found: C, $51.89 \% ; \mathrm{H}, 4.30 \%$; N, $10.63 \%$.

(2,8-Bis-trifluoromethylquinolin-4-yl)- $\mathbf{N}$-tert-butylethane-1,2-diamine (4). Yield: 56 \%, m.p. $90{ }^{\circ} \mathrm{C}$; IR (Neat, $\mathrm{cm}^{-1}$ ): 3350, 2955, 2830, 1598, 1550, 1445, 1315; LCMS/MS: m/z $380(\mathrm{M}+1)$; ${ }^{1} \mathrm{H}$ NMR $\left(\mathrm{CDCl}_{3}\right): \delta 8.05\left(\mathrm{~d}, \mathrm{~J}=7.2 \mathrm{~Hz}, 1 \mathrm{H},-\mathrm{C} \underline{\mathrm{H}}-\mathrm{C}-\mathrm{CF}_{3}\right), 8.00(\mathrm{~d}, \mathrm{~J}=8.4 \mathrm{~Hz}, 1 \mathrm{H}), 7.56(\mathrm{t}, \mathrm{J}=7.9$ $\mathrm{Hz}, 1 \mathrm{H}), 6.72(\mathrm{~s}, 1 \mathrm{H}), 6.55(\mathrm{~s}, 1 \mathrm{H},-\mathrm{NH}), 3.40\left(\mathrm{~m}, 2 \mathrm{H},-\mathrm{HNC}_{2}-\right), 2.82\left(\mathrm{~m}, 2 \mathrm{H},-\underline{\mathrm{H}}_{2} \mathrm{NH}-\right), 1.20$ (m, 9H, $\left.-\mathrm{C}\left(\mathrm{CH}_{3}\right)_{3}\right)$; Anal. Calcd for $\mathrm{C}_{17} \mathrm{H}_{19} \mathrm{~F}_{6} \mathrm{~N}_{3}$ (379.15): C, $53.83 \%$; H, $5.05 \%$; N, $11.08 \%$. Found: C, $53.85 \%$;, $5.00 \%$;, $11.00 \%$.

General method for the synthesis of bisquinoline derivatives 5-10. A solution of 2,8bis(trifluoromethyl)-4-chloroquinoline $(10 \mathrm{mmol} ; 3.0 \mathrm{~g})$, triethylamine $(10 \mathrm{mmol} ; 1.0 \mathrm{~g})$ and diamine $(5 \mathrm{mmol})$ in $\mathrm{N}$-methylpyrrolidinone $(10 \mathrm{ml})$ was heated to reflux for $10-24 \mathrm{~h}$ under a positive $\mathrm{N}_{2}$ pressure. After the reaction mixture was cooled to room temperature, ethyl acetate $(15 \mathrm{ml})$ and water $(15 \mathrm{ml})$ were added with stirring, and the resulting solid was filtered and washed with water to provide the derivatives 5-10. In all the cases, cooling of this two-phase mixture was required to induce precipitation of the product.

$N^{1}, N^{2}$-Bis-(2,8-bis-trifluoromethyl-quinolin-4-yl)-ethane-1,2-diamine (5). Yield: $41 \%$, m.p. 83-84 ${ }^{\circ} \mathrm{C}$; IR $\left(\mathrm{KBr}, \mathrm{cm}^{-1}\right): 3439,2918,1621,1597,1582,1541,1467,1310,1181$; LCMS/MS: $\mathrm{m} / \mathrm{z} 587(\mathrm{M}+1) ;{ }^{1} \mathrm{H} \mathrm{NMR}\left(\mathrm{CDCl}_{3}\right): \delta 8.36\left(\mathrm{~d}, \mathrm{~J}=8.4 \mathrm{~Hz}, 2 \mathrm{H},-\mathrm{C} \underline{\mathrm{H}}-\mathrm{C}-\mathrm{CF}_{3}\right), 8.03(\mathrm{~d}, \mathrm{~J}=7.1 \mathrm{~Hz}$,

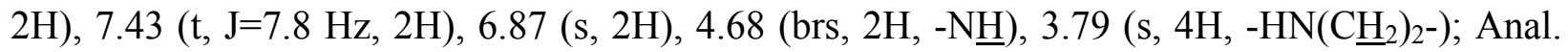
Calcd for $\mathrm{C}_{24} \mathrm{H}_{14} \mathrm{~F}_{12} \mathrm{~N}_{4}$ (586.38): C, $49.16 \%$; H, $2.41 \%$; N, $9.55 \%$. Found: C, $49.10 \%$; H, 2.40 $\% ; \mathrm{N}, 9.50$.

$\boldsymbol{N}^{\mathbf{1}}, \boldsymbol{N}^{\mathbf{7}}$-Bis-(2,8-bis-trifluoromethylquinolin-4-yl)-heptane-1,7-diamine (6). Yield: $45 \%$, m.p. $102{ }^{\circ} \mathrm{C}$; IR (KBr, cm $\left.{ }^{-1}\right): 3460,2939,2865,1597,1552,1477,1441,1313,1140$; LCMS/MS: $\mathrm{m} / \mathrm{z} 657(\mathrm{M}+1)$; ${ }^{1} \mathrm{H}$ NMR $\left(\mathrm{CDCl}_{3}\right): \delta 8.39\left(\mathrm{~d}, \mathrm{~J}=8.4 \mathrm{~Hz}, 2 \mathrm{H},-\mathrm{C} \underline{\mathrm{H}}-\mathrm{C}-\mathrm{CF}_{3}\right), 8.04(\mathrm{~d}, \mathrm{~J}=7.1 \mathrm{~Hz}, 2 \mathrm{H})$,

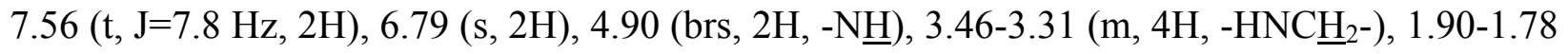
(m, 4H, - $\mathrm{HNCH}_{2} \mathrm{CH}_{2}-$ ), 1.52 (brs, 6H, - $\left(\mathrm{CH}_{2}\right)_{3}-$ ); Anal. Calcd for $\mathrm{C}_{29} \mathrm{H}_{24} \mathrm{~F}_{12} \mathrm{~N}_{4}$ (656.51): $\mathrm{C}, 53.05$ $\%$; H,3.68 \%; N, $8.53 \%$. Found: C, $53.00 \%$; $3.65 \%$; N, $8.50 \%$.

$\boldsymbol{N}^{\mathbf{1}}, \boldsymbol{N}^{\mathbf{9}}$-Bis-(2,8-bis-trifluoromethylquinolin-4-yl)-nonane-1,9-diamine (7). Yield: $38 \%$, m.p. $110^{\circ} \mathrm{C}$; IR $\left(\mathrm{KBr}, \mathrm{cm}^{-1}\right)$ : 3455, 2935, 2862, 1597, 1578, 1535; LCMS/MS: m/z 685 (M+1); ${ }^{1} \mathrm{H}$ NMR $\left(\mathrm{CDCl}_{3}\right): \delta 8.39\left(\mathrm{~d}, \mathrm{~J}=8.4 \mathrm{~Hz}, 2 \mathrm{H},-\mathrm{CH}-\mathrm{C}-\mathrm{CF}_{3}\right), 8.05(\mathrm{~d}, \mathrm{~J}=6.9 \mathrm{~Hz}, 2 \mathrm{H}), 7.57(\mathrm{t}, \mathrm{J}=7.7$ 
$\mathrm{Hz}, 2 \mathrm{H}), 6.79$ (s, 2H), 4.86 (brs, 2H, -Nㅍ), 3.40-3.30 (m, 4H, - $\mathrm{HNCH}_{2}-$ ), 1.76-1.74 (m, 4H, $\left.\mathrm{HNCH}_{2} \mathrm{CH}_{2}-\right), 1.42$ (brs, $\left.10 \mathrm{H},-\left(\mathrm{CH}_{2}\right)_{5}\right)^{-}$; Anal. Calcd for $\mathrm{C}_{31} \mathrm{H}_{28} \mathrm{~F}_{12} \mathrm{~N}_{4}$ (684.56): C, $54.39 \%$; $\mathrm{H}$, $4.12 \%$; N, $8.18 \%$. Found: C, $54.35 \%$; H, $4.10 \%$; N, $8.15 \%$.

$\boldsymbol{N}^{\mathbf{1}}, \boldsymbol{N}^{\mathbf{3}}$-Bis-(2,8-bis-trifluoromethylquinolin-4-yl)-propane-1,3-diamine (8). Yield: $40 \%$, m.p. 98-100 ${ }^{\circ} \mathrm{C}$; IR $\left(\mathrm{KBr}, \mathrm{cm}^{-1}\right)$ : 3448, 2930, 1586, 1540, 1472; LCMS/MS: $\mathrm{m} / \mathrm{z} 601(\mathrm{M}+1) ;{ }^{1} \mathrm{H}$ $\operatorname{NMR}\left(\mathrm{CDCl}_{3}\right): \delta 8.38\left(\mathrm{~d}, \mathrm{~J}=8.4 \mathrm{~Hz}, 2 \mathrm{H},-\mathrm{C} \underline{\mathrm{H}}-\mathrm{C}-\mathrm{CF}_{3}\right), 8.05(\mathrm{~d}, \mathrm{~J}=7.0 \mathrm{~Hz}, 2 \mathrm{H}), 7.55(\mathrm{t}, \mathrm{J}=7.8 \mathrm{~Hz}$,

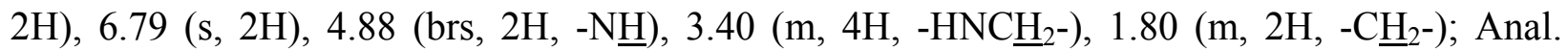
Calcd for $\mathrm{C}_{25} \mathrm{H}_{16} \mathrm{~F}_{12} \mathrm{~N}_{4}$ (600.12): C, $50.01 \%$; H, $2.69 \%$; N, $9.33 \%$. Found: C, $50.00 \%$; H, 2.60 $\%$; N, $9.30 \%$.

$\boldsymbol{N}^{\mathbf{1}}, \boldsymbol{N}^{\mathbf{4}}$-Bis-(2,8-bis-trifluoromethylquinolin-4-yl)-butane-1,4-diamine $\quad$ (9). Yield: $35 \%$, m.p. 106-108 ${ }^{\circ} \mathrm{C}$; IR $\left(\mathrm{KBr}, \mathrm{cm}^{-1}\right)$ : 3452, 2936, 2860, 1596, 1580, 1475; LCMS/MS: m/z 615 $(\mathrm{M}+1) ;{ }^{1} \mathrm{H}$ NMR $\left(\mathrm{CDCl}_{3}\right): \delta 8.39\left(\mathrm{~d}, \mathrm{~J}=8.4 \mathrm{~Hz}, 2 \mathrm{H},-\mathrm{C} \underline{\mathrm{H}}-\mathrm{C}-\mathrm{CF}_{3}\right), 8.00(\mathrm{~d}, \mathrm{~J}=7.0 \mathrm{~Hz}, 2 \mathrm{H}), 7.45$

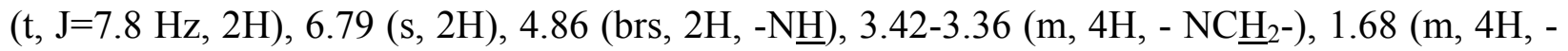
$\left.\left(\mathrm{C}_{2}\right)_{2}-\right)$; Anal. Calcd for $\mathrm{C}_{26} \mathrm{H}_{18} \mathrm{~F}_{12} \mathrm{~N}_{4}$ (614.13): C, $50.82 \%$; H, $2.95 \%$; N, $9.12 \%$, Found: C, $50.80 \%$; H, $2.98 \%$; N, $9.10 \%$.

$\boldsymbol{N}^{\mathbf{1}}, \boldsymbol{N}^{\mathbf{5}}$-Bis-(2,8-bis-trifluoromethylquinolin-4-yl)-pentane-1,5-diamine (10). Yield: $42 \%$, m.p. 96-98 ${ }^{\circ} \mathrm{C}$; IR $\left(\mathrm{KBr}, \mathrm{cm}^{-1}\right): 3458,2938,2862,1598,1582,1535,1470 ; \mathrm{CMS} / \mathrm{MS}: \mathrm{m} / \mathrm{z} 629$ $(\mathrm{M}+1) ;{ }^{1} \mathrm{H}$ NMR $\left(\mathrm{CDCl}_{3}\right): \delta 8.40\left(\mathrm{~d}, \mathrm{~J}=8.4 \mathrm{~Hz}, 2 \mathrm{H},-\mathrm{CH}-\mathrm{C}-\mathrm{CF}_{3}\right), 8.15(\mathrm{~d}, \mathrm{~J}=6.9 \mathrm{~Hz}, 2 \mathrm{H}), 7.56$

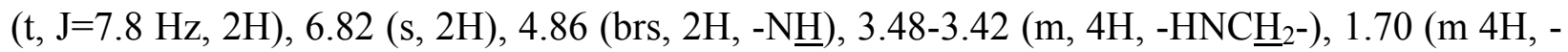
$\mathrm{HNCH}_{2} \mathrm{C}_{2^{-}}$), 1.34 (m, 2H, - $\underline{\mathrm{CH}}_{2}$ ); Anal. Calcd for ${ }_{27} \mathrm{H}_{20} \mathrm{~F}_{12} \mathrm{~N}_{4}(628.15): \mathrm{C}, 51.60 \%$; 3.21 $\%$; N, $8.92 \%$. Found: C, $51.50 \%$; H, $3.20 \%$; N, $8.90 \%$.

\section{Acknowledgements}

Authors are thankful to the Tuberculosis Antimicrobial Acquisition and Coordination Facility (TAACF), which provided antimycobacterial data through a research and development contract with the U.S. National Institute of Allergy and Infectious Diseases.

\section{References}

1. Corbett, E. L.; Watt, C. J.; Walker, N. Arch. Intern. Med. 2003, 163, 1009.

2. (a) Dye, C.; Scheele, S.; Dolin, P.; Pathania, V.; Raviglione, M. C. JAMA. 1999, 282, 677.

(b) World Health Organization. 2003. The WHO Global Tuberculosis Program. http://www.who.int/gtb/.

3. Houston, S.; Fanning, A. Drugs. 1994, 48, 689.

4. (a) Alland, D.; Kalkut, G. E.; Moss, A. R.; McAdam, R. A.; Hahn, J. A.; Bosworth, W.; Drucker, E.; Bloom, B. R. N. Engl. J. Med. 1994, 330, 1710. (b) Jacobs, R. F. Clin. Infect. Dis. 1994, 19, 1. (c) Weltman, A. C.; Rose, D. N. Arch. Intern. Med. 1994, 154, 2161. 
5. Lin, Y. M.; Flavin, M. T.; Cassidy, C. S.; Mar, A.; Chen, F. C. Bioorg. Med. Chem. Lett. 2001, 11, 2101.

6. Lin, Y. M.; Zhou, Y.; Flavin, M. T.; Zhou, L. M.; Nie, W.; Chen, F. C. Bioorg. Med. Chem. 2002, 10, 2795.

7. (a) Global Alliance for TB Drug Development. Scientific Blueprint for Tuberculosis Drug Development, 2001, (Suppl.): 1-52 (b) Smith, C. V.; Sharma, V.; Sacchettini, J. C. Tuberculosis. 2004, 84, 45. (c) Duncan, K.Tuberculosis. 2003, 83, 201.

8. Renau, T. E. ; Sanchez, J. P.; Shapiro, M. A.; Dever, J. A.; Gracheck, S. J.; Domagala, J. M. J. Med. Chem. 1995, 38, 2974.

9. Renau, T. E.; Sanchez, J. P.; Gage, J. W. ; Dever, J. A. ; Shapiro, M. A.; Gracheck, S. J.; Domagala, J. M. J. Med. Chem. 1996, 39, 729.

10. Berning, S. E. Drugs. 2001, 61, 9.

11. Mital, A; Ramachandran, U. Synthesis and biological evaluation of naphthoquinone derivatives as potent antimycobacterial agents. Paper orally presented by AM at the Singapore International Chemical Conference 4, SICC-4, 8-10 Dec, 2005, Singapore.

12. Ohnmacht, C. J.; Patel, A. R.; Lutz, R. E. J. Med. Chem. 1971, 14, 926.

13. Franzblau, S. G.; Witzig, R. S.; McLaughlin, J. C.; Torres, P.; Madico, G.; Hernandez, A.; Degnan, M. T.; Cook, M. B.; Quenzer, V. K.; Ferguson, R. M.; Gilman, R. H. J. Clin. Microb. 1998, 36, 362.

14. Collins, L. ; Franzblau, S. G. Antimicrob. Agents Chemother. 1997, 41, 1004. 